\title{
Shot noise near the unitary limit of a Kondo quantum dot
}

\author{
A. Golub \\ Department of Physics, \\ Ben-Gurion University of the Negev, Beer-Sheva, Israel
}

\begin{abstract}
We examine the statistics of current fluctuations in a junction with a quantum dot described by Kondo Hamiltonian. With the help of Keldysh technique we calculate shot noise as a function of applied voltage at zero temperature using the fix-point Hamiltonian. These calculations are complementary to similar ones performed earlier $([\mathbb{1}])$ in the mean field slave boson approximation. The results may be relevant for measurement of effective charge carriers.

PACS numbers: 72.10.Fk, 72.15.Qm, 73.63.Kv
\end{abstract}

Current noise has been studied extensively in the context of mesoscopic systems in the last couple of decades [2]. The zero-frequency noise out of equilibrium (shot noise) can yield information on charge fluctuations in such systems. We concentrate in the following on $T=0$, where the thermal noise vanishes and the only contribution to the current noise is shot noise. Due to the lack of a single accurate method that can describe the Kondo system out of equilibrium in all regimes, different methods need to span all physically relevant regimes 1]. Here we consider the regime around the strongly interacting fix point of Kondo Hamiltonian where shot-noise measurements can provide information about the charge of the carriers. The mean field slave boson approximation which is usually applied to describe this regime 1, 3, 4] involves numerical calculations and it yields to the more precise perturbation theory expansion in the least irrelevant perturbations.

We start with the Kondo Hamiltonian for quantum dot in the junction $H=H_{L}+H_{R}+H_{J}$ where

$$
H_{J}=\sum_{\alpha \alpha^{\prime} \sigma \sigma^{\prime}} J_{\alpha \alpha^{\prime}} c_{\alpha \sigma}^{\dagger}(0)\left(\frac{1}{4} \delta_{\sigma, \sigma^{\prime}}+\vec{S} \vec{s}_{\sigma \sigma^{\prime}}\right) c_{\alpha^{\prime} \sigma^{\prime}}(0)
$$

The first two terms correspond to non-interacting electrons in the two leads

$$
H_{L(R)}=\sum_{k, \sigma} \xi_{L(R) k} c_{L(R) \sigma, k}^{\dagger} c_{L(R) \sigma, k}
$$

where $c_{\alpha \sigma, k}, \xi_{\alpha k}$ are the electron field operator and the electron energy of a lead. Index $\alpha=L, R$ indicates left (right) lead. We assume that the leads are dc-biased by applied voltage $V$. Here $s$ is the one half spin matrix which acts on spin index of electron operators and $S$ is the spin operator of the dot. The potential scattering is represented by the first term in the brackets. We consider the case with the same dispersion law in both leads. Near the unitary limit we follow [5] and map the transport through the dot into a scattering problem. For this purpose, it is convenient to use the basis of $\mathrm{s}$ and $\mathrm{p}$ scattering states rather than that of the left-lead and rightlead states. At $V=0$ the p states are decoupled from the dot. For non-zero voltage the p-states are not decoupled, however, in the limit of low voltages $V<<T_{K}$ the s, p basis is important. Indeed, in this regime the dot is described by strongly interacting fix-point with many body state $(b)$. Before scattering the phases of both states, $b$ and $\mathrm{s}$-state, coincide, while passing the scattering region $b$ state acquires only an extra phase $\pi$ compared to that of s-state. Then the fix-point Hamiltonian can be written $[5,6,7]$ in the new basis $\left(b_{\sigma, k}, a_{\sigma, k}\right)$ (here $a$ stands for p-states) as $H=H_{0}+H_{s}+H_{i n t}$

$$
\begin{aligned}
H_{0} & =\sum_{k, \sigma} \xi_{k}\left(b_{\sigma, k}^{\dagger} b_{\sigma, k} a_{\sigma, k}^{\dagger} a_{\sigma, k}\right) \\
& +\frac{V}{2} \sum_{k, \sigma}\left(b_{\sigma, k}^{\dagger} a_{\sigma, k}+a_{\sigma, k}^{\dagger} b_{\sigma, k}\right) \\
H_{s} & =\frac{a}{\nu T_{K}} \sum_{k, k^{\prime}, \sigma}\left(\xi_{k}+\xi_{k^{\prime}}\right) b_{\sigma, k}^{\dagger} b_{\sigma, k^{\prime}} \\
H_{i n t} & =\frac{b}{\nu^{2} T_{K}} b_{\uparrow}^{\dagger} b_{\uparrow} b_{\downarrow}^{\dagger} b_{\downarrow}
\end{aligned}
$$

where $b=2 a$ and $a=1 /(2 \pi)[\underline{6}]$ and $b_{\uparrow}=\sum_{k} b_{\uparrow, k}$. The Kondo temperature $T_{K}$ is the only energy scale of the fixed-point Hamiltonian.

Current noise is defined as

$$
S(t) \equiv<I(t) I(0)>-<I>^{2},
$$

where the current operator requires a form

$$
\begin{aligned}
I= & \frac{2 e^{2} V}{h}-\frac{i e}{\hbar} \frac{a}{2 \nu T_{K}} \sum_{k, k^{\prime}, \sigma}\left(\xi_{k}+\xi_{k^{\prime}}\right) \\
& \left(b_{\sigma, k^{\prime}}^{\dagger} a_{\sigma, k}-a_{\sigma, k}^{\dagger} b_{\sigma, k^{\prime}}\right) \\
+ & \frac{i e}{\hbar} \frac{b}{2 \nu^{2} T_{K}} \sum_{\sigma}\left(b_{\sigma}^{\dagger} a_{\sigma}-a_{\sigma}^{\dagger} b_{\sigma}\right) n_{\bar{\sigma}}
\end{aligned}
$$

and $n_{\sigma}=b_{\sigma}^{\dagger} b_{\sigma}, \bar{\sigma}=-\sigma$.

The noise power can be represented as a sum of two terms $S=S_{\text {scat }}+S_{\text {int }}$ related, respectively, to the scattering part of the Hamiltonian $H_{s}$ and to the interaction part $H_{\text {int }}$. 


$$
\begin{aligned}
S_{\text {scat }}\left(t, t^{\prime}\right)= & \frac{e^{2}}{\hbar} \frac{a^{2}}{4 \nu^{2} T_{K}^{2}} \sum_{k_{i}, \sigma_{i}}\left(\xi_{k}+\xi_{k_{1}}\right)\left(\xi_{k^{\prime}}\right. \\
& \left.+\xi_{k_{1}^{\prime}}\right)<\left(b_{\sigma, k_{1}}^{\dagger} a_{\sigma, k}-a_{\sigma, k}^{\dagger} b_{\sigma, k_{1}}\right)_{t} \\
& \left(b_{\sigma_{1}, k_{1}^{\prime}}^{\dagger} a_{\sigma_{1}, k^{\prime}}-a_{\sigma_{1}, k^{\prime}}^{\dagger} b_{\sigma_{1}, k_{1}^{\prime}}\right)_{t^{\prime}}> \\
S_{\text {int }}\left(t, t^{\prime}\right)= & \frac{e^{2}}{\hbar} \frac{b^{2}}{4 \nu^{4} T_{K}^{2}}<\left.\left(b_{\sigma}^{\dagger} a_{\sigma}-a_{\sigma}^{\dagger} b_{\sigma}\right) n_{\bar{\sigma}}\right|_{t}\left(b_{\sigma_{1}}^{\dagger} a_{\sigma_{1}}\right. \\
- & \left.a_{\sigma_{1}}^{\dagger} b_{\sigma_{1}}\right)\left.n_{\overline{\sigma_{1}}}\right|_{t^{\prime}}>
\end{aligned}
$$

Here subscripts $t, t^{\prime}$ represent time dependence of operators. We can write the right hand side of $S_{\text {scat }}$ and $S_{\text {int }}$ as a time ordering product in Keldysh space. (This can be simply done by assigning Keldysh indices 2 and 1 to operators that depends on $t, t^{\prime}$, correspondingly. Then it is possible to express the result in terms of Green's functions of $H_{0}$ Hamiltonian. Let us perform the Fourier transform of equations( 78 ). Then for zero frequency noise (after summing over spin projections) we immediately get

$$
\begin{aligned}
S_{\text {scat }}= & \frac{e^{2}}{\hbar} \frac{a^{2}}{\nu^{2} T_{K}^{2}} \int \frac{d \omega}{2 \pi} \sum_{k, k_{1}}\left(\xi_{k}+\xi_{k_{1}}\right)^{2}\left[g_{d}^{21}\left(k_{1} \omega\right) g_{d}^{12}(k \omega)\right. \\
- & \left.g_{o f f}^{21}\left(k_{1} \omega\right) g_{o f f}^{12}(k \omega)\right] \\
S_{\text {int }}= & \frac{e^{2}}{\hbar} \frac{b^{2}}{T_{K}^{2}} \int \frac{d \omega_{1} d \omega_{2} d \omega_{3}}{(2 \pi)^{3}}\left[\left(g_{d}^{21}\left(\omega_{1}\right) g_{d}^{12}\left(\omega_{2}\right)-\right.\right. \\
& \left.g_{o f f}^{21}\left(\omega_{1}\right) g_{o f f}^{12}\left(\omega_{2}\right)\right) g_{d}^{21}\left(\omega_{3}\right) g_{d}^{12}\left(\omega_{1}-\omega_{2}+\omega_{3}\right)- \\
& \frac{1}{2}\left(g_{o f f}^{21}\left(\omega_{1}\right) g_{d}^{12}\left(\omega_{2}\right)-g_{d}^{21}\left(\omega_{1}\right) g_{o f f}^{12}\left(\omega_{2}\right)\right) \\
& \left(g_{d}^{21}\left(\omega_{3}\right) g_{o f f}^{12}\left(\omega_{1}-\omega_{2}+\omega_{3}\right)\right. \\
- & \left.g_{o f f}^{21}\left(\omega_{3}\right) g_{d}^{12}\left(\omega_{1}-\omega_{2}+\omega_{3}\right)\right)
\end{aligned}
$$

Green functions in the space of $a, b$ states can be written as a matrix $g$ with diagonal and off-diagonal entries each being Keldysh matrices.

$$
\begin{gathered}
g_{d}^{i j}\left(t, t^{\prime}\right)=-i<T b^{i}(t) b^{\dagger j}\left(t^{\prime}\right)> \\
g_{o f f}^{i j}\left(t, t^{\prime}\right)=-i<T b^{i}(t) a^{\dagger j}\left(t^{\prime}\right)>
\end{gathered}
$$

Explicitly, they acquire a form

$$
\begin{aligned}
g^{12}(k \omega)= & -f\left(\xi_{k}\right)\left(g^{R}(k \omega)-g^{A}(k \omega)\right) \\
g^{21}(k \omega)= & \left(1-f\left(\xi_{k}\right)\right)\left(g^{R}(k \omega)-g^{A}(k \omega)\right) \\
g^{R}(k \omega)- & g^{A}(k \omega)=-\pi i\left[\delta\left(\omega-\xi_{k}-e V / 2\right)+\right. \\
& \left.\delta\left(\omega-\xi_{k}+e V / 2\right)\right] \hat{I}+ \\
& {\left[\delta\left(\omega-\xi_{k}-e V / 2\right)-\delta\left(\omega-\xi_{k}+e V / 2\right)\right] \hat{\tau_{x}} } \\
g^{i, j}(\omega)= & \int d \xi_{k} g^{i, j}(k \omega) \\
g_{o f f}^{12}(\omega)= & g_{o f f}^{21}(\omega)=\pi i[f(\omega-e V / 2)-f(\omega+e V / 2)] \\
g_{d}^{12}(\omega)= & g_{d}^{21}(\omega)+2 \pi i \\
= & \pi i[f(\omega-e V / 2)+f(\omega+e V / 2)]
\end{aligned}
$$

With the help of these functions we get:

$$
\begin{aligned}
S_{\text {scat }}= & \frac{e^{2}}{h} \frac{a^{2} 8 \pi^{2}}{T_{K}^{2}} \int d \omega \omega^{2}\left[f\left(\omega-\frac{e V}{2}\right)\left(1-f\left(\omega+\frac{e V}{2}\right)\right)\right. \\
+ & f\left(\omega+\frac{e V}{2}\right)\left(1-f\left(\omega-\frac{e V}{2}\right)\right)= \\
= & \frac{2 e^{2}}{h}\left(\frac{a^{2} \pi^{2}}{3}\right) V \frac{(e V)^{2}}{T_{K}^{2}} \\
S_{i n t}= & \frac{e^{2}}{\hbar} \frac{\pi b^{2}}{8 T_{K}^{2}} \int d \omega_{1} d \omega_{2} d \omega_{3}\left[\left(2-F_{+}\left(\omega_{1}\right)\right) F_{+}\left(\omega_{2}\right)\right. \\
+ & \left.F_{-}\left(\omega_{1}\right) F_{-}\left(\omega_{2}\right)\right)\left(2-F_{+}\left(\omega_{3}\right)\right) F_{+}\left(\omega_{1}-\omega_{2}+\omega_{3}\right)+ \\
& \frac{1}{2}\left(\left(2-F_{+}\left(\omega_{1}\right)\right) F_{-}\left(\omega_{2}\right)+F_{-}\left(\omega_{1}\right) F_{+}\left(\omega_{2}\right)\right)((2 \\
- & \left.F_{+}\left(\omega_{3}\right)\right) F_{-}\left(\omega_{1}-\omega_{2}+\omega_{3}\right)+ \\
& \left.\left.F_{-}\left(\omega_{3}\right) F_{+}\left(\omega_{1}-\omega_{2}+\omega_{3}\right)\right)\right]
\end{aligned}
$$

where $F_{ \pm}(\omega)=f(\omega-e V / 2) \pm f(\omega+e V / 2)$.

Performing the triple integration (at $T \rightarrow 0$ ) we obtain part of the noise $S_{\text {int }}$ which is induced by interaction Hamiltonian $H_{\text {int }}$. Thus the total shot noise power in the limit of small voltages $\mathrm{eV}<<T_{K}$ acquires a form

$$
S_{i n t}=\frac{e^{2} V}{h} \pi^{2}\left(\frac{3}{2} b^{2}+\frac{2 a^{2}}{3}\right) \frac{(e V)^{2}}{T_{K}^{2}}
$$

For completeness we also calculate nonlinear conductance [5, 6]. Similarly to the noise the current consist of to parts $j=j_{\text {scat }}+j_{\text {int }}$, where $j_{\text {scat }}$ is the scattering part

$$
j_{\text {scat }}=\frac{e}{\hbar}<\hat{T}\left[H_{s}, N_{R}\right]_{t}^{1} \int d t^{\prime} \sigma_{z}^{i} H_{s}^{i}\left(t^{\prime}\right)>
$$

and $j_{\text {int }}$ interacting part

$$
j_{\text {int }}=\frac{e}{\hbar}<\hat{T}\left[H_{i n t}, N_{R}\right]_{t}^{1} \int d t^{\prime} \sigma_{z}^{i} H_{i n t}^{i}\left(t^{\prime}\right)>
$$

Here $\hat{T}$ represents time-ordering operator. We also explicitly introduced Keldysh indices $i=1,2$ and defined $\sigma^{1}=1, \sigma^{2}=-1$.

Thus for the scattering part of the current we obtain

$$
\begin{aligned}
j_{\text {scat }} & =\frac{e}{\hbar} \frac{a^{2}}{\nu^{2} T_{K}^{2}} \int \frac{d \omega}{2 \pi} \sum_{k, k_{1}}\left(\xi_{k}+\xi_{k_{1}}\right)^{2}\left(f\left(\xi_{k}\right)\right. \\
& \left.-f\left(\xi_{k_{1}}\right)\right) g_{d}^{d i f}\left(k_{1} \omega\right) g_{o f f}^{d i f}(k \omega)
\end{aligned}
$$

where $g^{\text {dif }}=g^{R}-g^{A}$. The frequency integration and momentum summation can be easily done with the result:

$$
j_{\text {scat }}=\frac{2 e^{2}}{h} \frac{a^{2} \pi^{2}}{3}\left(\frac{e V}{T_{K}}\right)^{2} V
$$

For interacting part we have

$$
j_{\text {int }}=\frac{2 e^{2}}{h} \frac{b^{2} \pi^{2}}{3} \frac{5}{4}\left(\frac{e V}{T_{K}}\right)^{2} V
$$


Thus total nonlinear conductance at zero temperature and in the case of small voltages $\mathrm{eV}<<T_{K}$ acquires a form

$$
\sigma=\frac{2 e^{2}}{h}\left[1-\pi^{2}\left(a^{2}+b^{2} \frac{5}{4}\right)\left(\frac{e V}{T_{K}}\right)^{2}\right]
$$

This result is in agreement with that obtained in [6].

This work was initiated by the problem of effective charge of carriers, the problem which was put forward by Y. Oreg and with whom (and also with E. Sela) the author has been communicated. Their interests in the noise calculations at the unitary limit of Kondo dot has stimulated this work. I would like to thank them both for discussions.
[1] Y. Meir, A. Golub, Phys. Rev. Lett. 88, 116802 (2002).

[2] Ya.M. Blanter and M. Buttiker, Phys. Rep. 336, 1 (2000).

[3] R. López, R. Aguado, G. Platero Phys. Rev. B 69, 235305 (2004)

[4] A.C. Hewson, The Kondo Problem to Heavy Fermions (Cambridge University Press, 1993).

[5] A. Kaminski, Yu.V. Nazarov, L.I. Glazman, Phys. Rev. B 62, 8154 (2000).

[6] M. Pustilnik and L. I. Glazman cond-mat/0501007

[7] I. Affleck, Nucl. Phys. B 336, 517 (1990). 